\title{
Theory of Thermodynamic Magnetic Oscillations in
}

\section{Quasi-One-Dimensional Conductors}

\author{
Victor M. Yakovenkot \\ Serin Physics Laboratory, Rutgers University, P.O.Box 849, Piscataway, NJ 08855
}

(Received 1992)

\begin{abstract}
The second order correction to free energy due to the interaction between electrons is calculated for a quasi-one-dimensional conductor exposed to a magnetic field perpendicular to the chains. It is found that specific heat, magnetization and torque oscillate when the magnetic field is rotated in the plane perpendicular to the chains or when the magnitude of magnetic filed is changed. This new mechanism of thermodynamic magnetic oscillations in metals, which is not related to the presence of any closed electron orbits, is applied to explain behavior of the organic conductor $(\mathrm{TMTSF})_{2} \mathrm{ClO}_{4}$.
\end{abstract}

PACS numbers: 71.25.Hc, 71.10.+x, 74.70.Kn, 75.20.En

Typeset Using REVTEX 
In a recent study [1] of the quasi-one-dimensional conductor (TMTSF) ${ }_{2} \mathrm{ClO}_{4}$, a new physical effect has been found. In this experiment, a magnetic field was rotated in the plane perpendicular to the conducting chains and the torque along the chains was measured. The torque, as a function of the angle $\theta$ between the magnetic field and the $c$-axis, was observed to exhibit oscillations approximately periodic in $\tan \theta$. Since the torque is a derivative of the free energy $F$ with respect to $\theta$, then $F$ must also oscillate upon rotation of the magnetic field. In earlier experiments, magnetization [2] and specific heat [3] were measured to be oscillating functions of the magnetic field magnitude (the so called "fast" oscillations). Such angular and fast thermodynamic magnetic oscillations were totally unexpected theoretically for a quasi-one-dimensional metal. In momentum space, an electron in a magnetic field moves along an orbit obtained as the intersection of a constant energy surface and a plane perpendicular to the magnetic field. Magnetic oscillations of the free energy (de Haasvan Alfven effect) are known in the situation when the orbits at the Fermi surface are closed. In this case, the orbits are quantized, the energy spectrum is discrete and the oscillations in $F$ are due to the Landau levels crossing the Fermi level. However, in a quasi-one-dimensional conductor, the Fermi surface consists of two slightly warped planes perpendicular to the direction of the chains. Thus, if a magnetic field has been applied perpendicular to the chains, all electron orbits at the Fermi surface are open, the energy spectrum is continuous and no oscillations in $F$ are expected for non-interacting electrons [4]. In the present paper, the lowest order correction to $F$ due to the interaction between electrons is calculated for a quasi-one-dimensional conductor and is shown to exhibit angular and fast magnetic oscillations.

The phenomenon of angular oscillations in a quasi-one-dimensional conductor was predicted theoretically by Lebed [5] who calculated the oscillations of the magnetic-field-induced spin-density-wave (FISDW) transition temperature. Experiment [6] did not reveal these oscillations, however, the studied range of angles might not have been big enough to see the first oscillation [1]. The angular oscillations in the conductivity were predicted [7] and observed [8.9.1]. Two other models for the oscillations in the conductivity were later suggested [10]. 
None of mentioned theoretical papers considered the oscillations of free energy. Recently, the contribution of the FISDW order parameter fluctuations to $F$ has been calculated and been shown to exhibit angular oscillations [11]. In the present paper, the approach of Ref. [5] and [7] is used to calculate the lowest order correction to $F$. This theory is applicable in the metallic phase sufficiently far from the phase boundary, while theory [11] is applicable in the vicinity of the phase transition to the FISDW state.

Let us consider a square lattice of parallel chains with a spacing $b$ between the chains, $x$-axis parallel the chains. The electron hopping integrals between the nearest chains in the $y$ and $z$ directions are equal to $t_{b}$ and $t_{c}$, respectively; $t_{c} \ll t_{b} \ll E_{F}$, where $E_{F}$ is the Fermi energy. Magnetic field $H$ is applied in the $(y, z)$ plane at an angle $\theta$ measured from $z$-axis. The electron Hamiltonian without the interaction term can be written as [12]:

$$
\begin{aligned}
& \hat{H}_{\alpha \sigma}=-\alpha i v_{F} \partial_{x}+2 t_{b} \cos \left(k_{y}-e b H x \cos \theta / c\right) \\
& +2 t_{c} \cos \left(k_{z}-e b H x \sin \theta / c\right)-\mu_{B} H \sigma,
\end{aligned}
$$

where the index $\alpha= \pm$ labels electrons whose momenta along the chains are close to $\pm k_{F}$, $v_{F}$ and $k_{F}$ are the Fermi velocity and the Fermi momentum, $k_{y}$ and $k_{z}$ are momenta along the $y$ - and $z$-axes, $\sigma$ is the spin index, $\mu_{B}$ is the Bohr magneton, $e$ is the electron charge, $c$ is the velocity of light and $\hbar \equiv 1$. The two cosine terms in (1), which describe electron motion perpendicular to the chains, become in magnetic field two periodic potentials along the chains. Their periods are commensurate at rational values of $\tan \theta$. This effect is responsible for the angular oscillations of free energy.

The lowest order non-trivial correction to the free energy $\Delta F$ due to the interaction between electrons is given by the diagram shown in Fig. 1. Using Green functions, derived from Eq. (11) [12], one can find the following expression for the free energy per electron:

$$
\begin{aligned}
& \Delta F=-\frac{2 \pi^{3} g^{2} T^{3}}{\varepsilon_{F} \Omega} \int_{0}^{\infty} d x \frac{\cosh (\tau x)}{\sinh ^{3}(\tau x)} f_{y}(x) f_{z}(x), \\
& f_{y}(x)=\left\langle J_{0}^{2}\left(V_{b} \sin (x \cos \theta) \sin k / \cos \theta\right)\right\rangle_{k} \\
& f_{z}(x)=\left\langle J_{0}^{2}\left(V_{c} \sin (x \sin \theta) \sin k / \sin \theta\right)\right\rangle_{k} .
\end{aligned}
$$


Here $\varepsilon_{F} \equiv v_{F} k_{F}, T$ is the temperature, $\Omega=e b v_{F} H / c$ is the characteristic energy of magnetic field, $\tau=4 \pi T / \Omega, V_{b(c)}=8 t_{b(c)} / \Omega, J_{0}$ is the Bessel function and $\langle\ldots\rangle_{k}$ means the averaging over $k . g^{2}=\left(g_{2}-g_{1}\right)^{2}+g_{2}^{2}$, where $g_{1}$ and $g_{2}$ are dimensionless interaction constants between electrons, normalized in such a way that the mean-field SDW transition temperature is proportional to $\exp \left(-1 / g_{2}\right)$. Another contribution to $\Delta F$, which comes from spin reversal scattering, contains an additional factor $\cos \left(4 \mu_{B} H x / \Omega\right)$ under the integral in Eq. (目) and $g_{1}^{2}$ appears instead of $g^{2}$. This contribution is more complex and will not be studied here.

Integral (2) is divergent at small $x$. However, observable quantities considered below are given by convergent integrals. The correction to the specific heat per electron, derived from Eq. (2), takes the form:

$\Delta C=-\frac{3}{4} g^{2} C_{0} \int_{0}^{\infty} d x\left(\frac{x^{2}}{\sinh ^{2} x}\right)^{\prime \prime \prime} f_{y}\left(\frac{x}{\tau}\right) f_{z}\left(\frac{x}{\tau}\right)$,

where the primes denote the third derivative with respect to $x$ and $C_{0}=\pi^{2} T / 3 \varepsilon_{F}$ is the specific heat of non-interacting electrons. In Fig. 2, $\Delta C(\theta)$ is shown for three different values of $\tau(0.1,0.2$ and 0.4$)$ at $V_{b}=20$ and $V_{c}=2 . \Delta C$ exhibits sharp peaks when $\tan \theta$ attains rational values, which is where the periods of the functions $f_{y}(x)$ and $f_{z}(x)$ in (5) become commensurate. The widths of the peaks go to zero when $T \rightarrow 0$ and the curve becomes fractal. Each peak is split because the term $\left(x^{2} / \sinh ^{2} x\right)^{\prime \prime \prime}$ in (5) oscillates once over the range $[0, \infty]$. This splitting vanishes as $T \rightarrow 0$. It follows from (3i) and (4⿴囗十) that the transformation $\theta \rightarrow 45^{\circ}-\theta$ interchanges $V_{b}$ and $V_{c}$ in (5), having small effect on the plots $\Delta C(\theta)$ in Fig. 2, despite the employed values of $V_{b}$ and $V_{c}$ differ by an order of magnitude. This means that $\Delta C$ does not depend qualitatively on the precise values of $V_{b}$ and $V_{c}$ if they are greater than 2. According to Ref. [13], in (TMTSF) ${ }_{2} \mathrm{ClO}_{4}, \Omega / H=1.8 \mathrm{~K} / \mathrm{T}$, but due to the unit cell doubling the two times bigger value should be used. Thus, the field e.g. $5 \mathrm{~T}$ corresponds to $\Omega=18 \mathrm{~K}$ and the values of the parameters $\tau$ and $V$ in Fig. 2 correspond to $T=0.14 \mathrm{~K}, 0.29 \mathrm{~K}, 0.57 \mathrm{~K}, t_{b}=45 \mathrm{~K}$ and $t_{c}=4.5 \mathrm{~K}$. The dependence of specific heat on the orientation of the magnetic field has not been measured yet experimentally.

The correction to the magnetization per electron along the $z$-axis $\Delta M_{z}=-\partial \Delta F / \partial H_{z}$, 
where $H_{z}=H \cos \theta$, is given by the following expression:

$\Delta M_{z}=\frac{g^{2} t_{b} \Omega}{2 \varepsilon_{F} H} \int_{0}^{\infty} d x \frac{\cosh \left(\frac{x \tau}{\cos \theta}\right)(x \cos x-\sin x)}{\left(\sinh \left(\frac{x \tau}{\cos \theta}\right) \frac{\cos \theta}{\tau}\right)^{3}}$

$\left\langle\sin k J_{1}\left(\frac{V_{b} \sin x \sin k}{\cos \theta}\right) J_{0}\left(\frac{V_{b} \sin x \sin k}{\cos \theta}\right)\right\rangle_{k} f_{z}\left(\frac{x}{\cos \theta}\right)$,

Unlike $\Delta C, \Delta M_{z}(\theta)$ has a limit of $T=0$ which is shown in Fig. 3 at $V_{b}=20$ and $V_{c}=2$. Note that the magnetic energy $H_{z} \Delta M_{z}$ may be much greater than the Zeeman energy $\left(\mu_{B} H\right)^{2} / \varepsilon_{F}$ and the orbital energy $\left(t_{b}^{2} \cos ^{2} \theta+t_{c}^{2} \sin ^{2} \theta\right) \Omega^{2} / 12 \varepsilon_{F}^{3}$ of the non-interacting electrons (the latter formula is a result beyond quasi-classics). The magnetization is found to be negative (diamagnetic) in agreement with the experiments in the high field reentrant metallic phase of $(\mathrm{TMTSF})_{2} \mathrm{ClO}_{4}$ [2]. The torque is determined by the expression $N_{x}=-\Delta M_{z} H \cos \theta+$ $\Delta M_{y} H \sin \theta$, where $\Delta M_{y}$ is given by Eq. (6) after interchanging $t_{b}$ with $t_{c}$ and $\cos \theta$ with $\sin \theta$. The torque is plotted in Fig. 3 at $\tau=0$ and $\tau=0.25$ together with an experimental curve taken at $T=0.4 \mathrm{~K}, H=5 \mathrm{~T}[14]$. Using the cited above value of $\Omega / H$, one finds $\tau=0.28$ in the experiment, close to the value employed for the theoretical curve c. There is an overall qualitative resemblance between the theoretical and the experimental curves. For a detailed comparison one should take into account the different spacings of the chains along the $y$ - and $z$-axes and the triclinic structure of the real material. Note that, with few exceptions, the peaks in Fig. 3 appear when $\tan \theta$ is integer, not fractional as in Fig. 2, in agreement with the experimental observation [1]. This happens because at $\tau=0$ the aperiodic function of $x$ under the integral (6) decreases rapidly when $x \geq 2 \pi$, while the last function in (6) has the (longer) period of the order of $\pi / \tan \theta$, thus the resonances cannot take place at small $\tan \theta$.

In Fig. $4, \Delta M_{z}$ (6) at $\theta=0$ is plotted vs the variable $1 / V_{b}$, which is proportional to the magnetic field. $\Delta M_{z}$ exhibits oscillations periodic in inverse magnetic field, which may correspond to the fast oscillations of magnetization observed in Ref. [2]. The period of the oscillations $\Delta(1 / H)$ is determined by the condition $\Delta V_{b}=\pi$ and equals $\pi e b v_{F} / 8 c t_{b}$. The numbers of oscillations in Fig. 4 are from 3 to 15 . Using the experimental value $255 \mathrm{~T}$ for the frequency of the fast oscillations [2] one finds $t_{b}=180 \mathrm{~K}$. A similar mechanism for the 
fast oscillations of conductivity was studied in Ref. [15]. However, we have not found these oscillations in specific heat (5).

Another possible explanation of the fast oscillations [16] takes into account that the unit cell of (TMTSF) $)_{2} \mathrm{ClO}_{4}$ is doubled in the $y$ direction due to a crystal superstructure, thus the energy of a chain staggers by the value $\pm \kappa$ along the $y$-axis. Suppose, that $\kappa \gg$ $t_{b}$. In this case, the conduction band is split into two bands with the Fermi momenta $k_{F} \pm \kappa / v_{F}$, and the effective transverse hopping integral $t_{b}^{*}$ is equal to $t_{b}^{2} / 2 \kappa$. In one band, the electron wave functions are predominant on even chains; in another band, on odd chains. Interaction between the electrons belonging to the same band reproduces the formulas given above with $t_{b}^{*}$ substituted for $t_{b}$ and the spacing along $y$-axis doubled. This prescription immediately explains why in experiments [1,9] the angular oscillations were found only at even, instead of all, integer values of $\tan \theta$ defined with respect to the original lattice without superstructure. In addition, the interaction of the electrons belonging to the different bands gives the following contribution to the free energy (at $\theta=0)$ :

$$
\begin{aligned}
& \Delta^{\prime} F=-\frac{2 \pi^{3} g^{2} T^{3} t_{b}^{4}}{\varepsilon_{F} \Omega \kappa^{4}} \int_{0}^{\infty} d x \frac{\cosh \left(x \tau^{\prime}\right)}{\sinh ^{3}\left(x \tau^{\prime}\right)}\left\langle J_{0}^{2}\left(V_{c} x \sin p\right)\right\rangle_{p} \\
& \left\langle\left[\left(1+2 \cos ^{2} x\right) J_{0}^{2}\left(V_{b}^{*} \sin x \sin k\right)-J_{1}^{2}\left(V_{b}^{*} \sin x \sin k\right)\right]\right. \\
& \sin ^{2} k \cos (2 R x)+4 J_{0}\left(V_{b}^{*} \sin x \sin k\right) J_{1}\left(V_{b}^{*} \sin x \sin k\right) \\
& \sin k \cos x \sin (2 R x)\rangle_{k},
\end{aligned}
$$

where $V_{b}^{*}=4 t_{b}^{*} / \Omega, \tau^{\prime}=\tau / 2$ and $R=2 \kappa / \Omega$. The correction to the specific heat $\Delta^{\prime} C$ is obtained from (7) in the same way as Eq. (5) is obtained from Eq. (2) and is plotted vs $R$ in Fig. 5. $\Delta^{\prime} C$ exhibits oscillations periodic in inverse magnetic field which may correspond to the fast oscillations of specific heat found in Ref. [3]. The period of the oscillations is determined by the condition $\Delta R=1$ and is given by $\Delta(1 / H)=e b v_{F} / 2 c \kappa$, which corrects by a factor of 2 the expression found in Ref. [16]. The numbers of oscillations in Fig. 5 are from 2 to 40. Using the experimental frequency of the fast oscillations 257T [3] one finds $2 \kappa=460 \mathrm{~K}$. Analogous oscillations should also exist in magnetization, which can be derived from Eq. (7) as well. If, on the other hand, the opposite limit $\kappa \leq t_{b}$ holds, then, in order 
to explain why the odd peaks are missing in experiments [1.9], it is necessary to conclude that the field $H_{z}$ at $H=5 \mathrm{~T}$ is below the magnetic breakdown field $H_{0}=\kappa^{2} c / t_{b} e b v_{F}$. In this case, the mechanism described above still works qualitatively but with the frequency of the fast oscillations proportional to $t_{b}$ instead of $\kappa$. If the odd peaks appear experimentally in higher fields, this can give the estimates of $H_{0}$ and $\kappa$.

Fast oscillations were also observed in the material (TMTSF) ${ }_{2} \mathrm{PF}_{6}$, which does not have an anion superstructure [17]. This fact supports the first explanation of the fast oscillations which does not invoke the superstructure. On the other hand, these oscillations were observed thus far only in the FISDW phase in transport measurements. Thus, formally, the fast oscillations in (TMTSF $)_{2} \mathrm{PF}_{6}$ are outside the applicability range of presented theory and a definitive choice between two explanations cannot be made (see also Ref. [18]).

In conclusion, the second order correction to the free energy due to the interaction between electrons has been calculated for a quasi-one-dimensional metal. Angular and fast magnetic oscillations of specific heat, magnetization and torque were found in qualitative agreement with experiment. Two different mechanisms of the fast oscillations, related and unrelated to the specific crystal structure of (TMTSF $)_{2} \mathrm{ClO}_{4}$, were observed. Unlike in the standard theory of metals, the oscillations are completely due to the interactions between electrons and cannot be interpreted in terms of the presence of some closed electron orbits. The oscillations involve the energy scales of the cyclotron frequency $\Omega$, the hopping integral $t_{b}$ or the anion superstructure splitting $\kappa$, which are much higher than the characteristic energy scale of the deviation from nesting and the FISDW transition temperature. That explains why the oscillations persist in the FISDW phase as observed experimentally.

The author thanks M. J. Naughton for sending torque data shown in Fig. 3; J. S. Brooks, who suggested to calculate specific heat; both of them and P. M. Chaikin, for discussions. This work was supported by the NSF Grant No. DMR 89-06958. 


\section{REFERENCES}

* On leave from L. D. Landau Institute for Theoretical Physics, Moscow, 117940, Russia.

1 M. J. Naughton et al., Phys. Rev. Lett. 67, 3712 (1991).

${ }^{2}$ X. Yan et al., Phys. Rev. B 36, 1799 (1987); R. V. Chamberlin et al., Phys. Rev. Lett. 60, 1189 (1988).

3 N. A. Fortune et al., Phys. Rev. Lett. 64, 2054 (1990).

${ }^{4}$ I. M. Lifshits, M. Ya. Azbel and M. I. Kaganov, Electron Theory of Metals (Consultants Bureau, N.Y., 1973).

5 A. G. Lebed, Pis'ma Zh. Exp. Teor. Fiz. 43, 137 (1986) [JETP Lett. 43, 174 (1986)].

${ }^{6}$ X. Yan et al., Solid State Commun. 66, 905 (1988).

7 A. Lebed and P. Bak, Phys. Rev. Lett. 63, 1315 (1989).

${ }^{8}$ G. S. Boebinger et al., Phys. Rev. Lett. 64, 591 (1990); M. J. Naughton et al., Mater. Res. Soc. Symp. Proc. 173, 257 (1990).

9 T. Osada et al., Phys. Rev. Lett. 66, 1525 (1991).

10 T. Osada, S. Kagoshima and N. Miura, Technical report of ISSP, Univ. of Tokyo, Ser. A, No. 2470 (1991); K. Maki, Phys. Rev. B 45, 5111 (1992).

11 A. Bjelič and K. Maki, Phys. Rev. B, to be published.

${ }^{12}$ L. P. Gor'kov and A. G. Lebed', J. Phys. (Paris) Lett. 45, L433 (1984).

${ }^{13}$ V. M. Yakovenko, Zh. Exp. Teor. Fiz. 93, 627 (1987) [Sov. Phys. JETP 66, 355 (1987)].

14 The author is grateful to M. J. Naughton for permission to publish the raw experimental torque data of Ref. [四]. The curve d in Fig. 3 actually represents the data between two main minima in the angle interval from $6^{\circ}$ to $-90^{\circ}$ mapped for the purpose of qualitative comparison to the interval $0^{\circ}$ to $90^{\circ}$. 
15 K. Yamaji, J. Phys. Soc. Jpn. 55, 1424 (1986).

16 S. A. Brazovskii and V. M. Yakovenko, Pis'ma Zh. Exp. Teor. Fiz. 43, 102 (1986) [JETP Lett. 43, $134(1986)]$.

17 J. P. Ulmet et al., J. Phys. (Paris) Lett. 46, L535 (1985); J. R. Cooper et al., Phys. Rev. Lett. 63, 1984 (1988); S. T. Hannahs et al., ibid. 63, 1988 (1988); W. Kang et al., Phys. Rev. B, to be published.

18 A. G. Lebed', Phys. Scripta 39, 386 (1991). 


\section{FIGURES}

FIG. 1. The second order correction to the free energy. The lines, labeled by + and - , represent Green functions of the electrons with momenta close to $\pm k_{F}$. The vertices represent the amplitudes of interaction between electrons.

FIG. 2. Normalized correction to specific heat $-\Delta C / 3 g^{2} C_{0}$ vs magnetic field rotation angle $\theta$. Parameter $\tau$ is equal to 0.1 (curve a), 0.2 (b) and 0.4 (c). The arrows at the top indicate the rational values of $\tan \theta$.

FIG. 3. Normalized magnetization $-16 \Delta M_{z} H \varepsilon_{F} / g^{2} \Omega^{2}$ (curve a) and normalized torque $16 N_{x} \varepsilon_{F} / g^{2} \Omega^{2}$ (curve b, $\tau=0$, and curve c, $\tau=0.25$ ) calculated as functions of $\theta$. The curve d represents the experimental torque data due to M. J. Naughton [14].

FIG. 4. Normalized magnetization $-16 \Delta M_{z} H \varepsilon_{F} / g^{2} \Omega^{2}$ at $T=0$ vs normalized magnetic field $1 / V_{b}=e b v_{F} H / 8 t_{b} c ; t_{c}=0$ in curve a and $t_{c}=0.1 t_{b}$ in curve b.

FIG. 5. Additional normalized correction to specific heat in the presence of the special crystal superstructure $-\Delta^{\prime} C \kappa^{4} / 6 g^{2} t^{4} C_{0}$ vs normalized inverse magnetic field $R=2 \kappa c / e b v_{F} H$. Parameters are: $t_{b}^{*}=\kappa / 8, t_{c}=\kappa / 80$ and $T=\kappa / 20 \pi^{2}$. 